\title{
Innermost stable circular orbit near dirty black holes in magnetic field and ultra-high-energy particle collisions
}

\author{
O. B. Zaslavskii ${ }^{1,2, a}$ \\ ${ }^{1}$ Department of Physics and Technology, Kharkov V.N. Karazin National University, 4 Svoboda Square, Kharkov 61022, Ukraine \\ ${ }^{2}$ Institute of Mathematics and Mechanics, Kazan Federal University, 18 Kremlyovskaya St., Kazan 420008, Russia
}

Received: 21 August 2014 / Accepted: 13 August 2015 / Published online: 3 September 2015

(C) The Author(s) 2015. This article is published with open access at Springerlink.com

\begin{abstract}
We consider the behavior of the innermost stable circular orbit (ISCO) in the magnetic field near "dirty" (surrounded by matter) axially symmetric black holes. The cases of near-extremal, extremal, and nonextremal black holes are analyzed. For nonrotating black holes, in the strong magnetic field ISCO approaches the horizon (when backreaction of the field on the geometry is neglected). Rotation destroys this phenomenon. The angular momentum and radius of ISCO look model-independent in the main approximation. We also study the collisions between two particles that results in the ultra-high energy $E_{\text {c.m. }}$ in the center-of-mass frame. Two scenarios are considered - when one particle moves on the nearhorizon ISCO or when collision occurs on the horizon, one particle having the energy and angular momentum typical of ISCO. If the magnetic field is strong enough and a black hole is slowly rotating, $E_{\text {c.m. }}$ can become arbitrarily large. The kinematics of the high-energy collision is discussed. As an example, we consider the magnetized Schwarzschild black hole for an arbitrary strength of the field (the Ernst solution). It is shown that backreaction of the magnetic field on the geometry can bound the growth of $E_{\mathrm{c} . \mathrm{m} .}$.
\end{abstract}

\section{Introduction}

The motion of particles in the vicinity of black holes is a subject that has been continuing to attract interest until recently. In doing so, a special role is played by circular orbits-see, e.g., Refs. [1,2] and references therein. Especially, this concerns an innermost stable circular orbit (ISCO). It is important in phenomena connected with accretion disc and properties of cosmic plasma [3,4]. Apart from astrophysics, such a kind of orbits possesses a number of nontrivial features and, therefore, is interesting from the theoretical viewpoint. In a classic paper [5] it was shown that in the extremal limit ISCO

\footnotetext{
a e-mail: zaslav@ukr.net
}

approaches the horizon. As a result, some subtleties arise here since the horizon is a lightlike surface, so a massive particle cannot lie within it exactly. Nowadays, near-horizon circular orbits for near-extremal and extremal rotating black holes are still a subject of debates [6-9].

Quite recently, a new circumstance came into play that makes the properties of ISCO important in a new context. Namely, it is the ISCO that turns out to be a natural venue for the realization of the so-called BSW effect. Several years ago, it was shown by Bañados, Silk, and West that if two particles collide near the black hole horizon of the extremal Kerr metric, their energy $E_{\text {c.m. }}$. in the center-of-mass $(\mathrm{CM})$ frame can grow unboundedly [10]. These findings stimulated further study of high-energy collisions near black holes. The validity of the BSW effect was extended to extremal and nonextremal more general black holes. It was also found that there exists a version of this effect near nonrotating electrically charged black holes [11]. Another version of ultra-high-energy collisions reveals itself in the magnetic field, even if a black hole is neutral, vacuum, and nonrotating, so it is described by the Schwarzschild metric [12]. Generalization to the case when the background is described by the Kerr metric was done in [13].

In the BSW effect, one of the colliding particles should be so-called critical. It means that the energy and the angular momentum (or electric charge) of this particle should be finetuned. In particular, the corresponding critical condition is realized with good accuracy if a particle moves on a circular orbit close to the horizon. Therefore, an ISCO can play a special role in ultra-high-energy collisions in astrophysical conditions. Without the magnetic field, this was considered in [14] for the Kerr black hole and in [15] for more general rotating black holes. Kinematically, the effect is achieved due to collision of a rapid typical so-called usual particle (without fine-tuning) and the slow fine-tuned particle on the ISCO [16] (see also below). 
In $[12,13]$ collisions were studied just near the ISCO in the magnetic field. In both cases, a black hole was taken to be a vacuum one. Meanwhile, in astrophysical conditions, black holes are surrounded by matter. By definition, such black holes are called "dirty", according to the terminology suggested in Ref. [17]. (We would like to stress that it is matter but not the electromagnetic field that makes a black hole dirty.)

The aim of our work is two-fold, since two different issues overlap here. The first one concerns the properties of ISCO near dirty black holes in a magnetic field, so both matter and the magnetic field are present. The second issue concerns the scenarios of high-energy particle collisions near such orbits. We derive general asymptotic formulas for the position of the ISCO in the magnetic field, which are used further for the evaluation of $E_{\mathrm{c} . \mathrm{m}}$. and examining of two scenarios of the BSW effect near ISCO.

In Refs. [12,13], it was assumed that the magnetic field is weak in the sense that backreaction of the magnetic field on the metric is negligible but, at the same time, it is strong in the sense that it affects he motion of test particles. Such a combination is self-consistent, since the dimensionless parameter $b$ that controls the magnetic field strength contains a large factor $q / m$, relevant for the motion of particles. Our approach is model-independent and is not restricted by some explicit background metric. Therefore, most of the formulas apply also to the metrics which are affected by the magnetic field. On the other hand, if the magnetic field is too strong, its backreaction on the metric can change the properties of $E_{\text {c.m. }}$ itself, as will be seen below. Thus we discuss two new features absent from previous works in the sense that both matter and magnetic field are taken into account in a modelindependent way.

It is worth noting that high-energy collisions in the magnetic field were studied also in another context, including scenarios not connected with ISCO-see [18-21].

In general, it is hard to find and analyze ISCO even in the Kerr or Kerr-Newman cases [1,2]. However, it is the proximity to the horizon that enables us to describe some properties of ISCO, even not specifying the metric (so we work in a model-independent way) and even with a magnetic field. This can be considered as one manifestation of universality of black hole physics.

The paper is organized as follows. In Sect. 2, the metric and equations of motion are presented. In Sect. 3, we give basic equations that determine ISCO. In Sect. 4, we consider ISCO in a magnetic field for near-extremal black holes and analyze the cases of small and large fields. In Sect. 5 the cases of nonrotating (but dirty) and slowly rotating black holes are discussed. As we have two small parameters (slow rotation and inverse field strength), we consider different relations between them separately. In Sect. 6 we show that if a black hole rotates, even in the limit of a strong magnetic field ISCO does not tend to the horizon radius. In Sect. 7, it is shown that for extremal nonrotating black holes, for large $b$, ISCO approaches the horizon radius. In Sect. 8, it is shown that this property is destroyed by rotation. In Sect. 9 , general formulas for $E_{\text {c.m. }}$ for particle collisions in the magnetic field are given. In Sect. 10, we find the velocity of a particle on ISCO and argue that a kinematic explanation of high-energy collisions is similar to that for the BSW effect [16]. In Sect. 11, we apply general formulas of collision to different black hole configurations and different scenarios. In Sect. 12, the exact solution of the Einstein-Maxwell equations (static Ernst black hole) is chosen as a background for collisions. This enables us to evaluate the role of backreaction of the magnetic field on $E_{\text {c.m. }}$. In Sect. 13, the main results are summarized. Some technical points connected with cumbersome formulas are put in the appendix.

Throughout the paper we use units in which the fundamental constants are $G=c=1$.

\section{Metric and equations of motion}

Let us consider the metric of the form

$\mathrm{d} s^{2}=-N^{2} \mathrm{~d} t^{2}+\frac{\mathrm{d} r^{2}}{A}+R^{2}(\mathrm{~d} \phi-\omega \mathrm{d} t)^{2}+g_{\theta} \mathrm{d} \theta^{2}$,

where the metric coefficients do not depend on $t$ and $\phi$. The horizon corresponds to $N=0$. We also assume that there is an electromagnetic field with the four-vector $A^{\mu}$ where the only nonvanishing component equals

$A^{\phi}=\frac{B}{2}$

In vacuum, this is an exact solution with $B=$ const [22]. We consider configuration with matter (in this sense a black hole is "dirty"), so in general $B$ may depend on $r$ and $\theta$.

Let us consider motion of test particles in this background. The kinematic momentum $p^{\mu}=m u^{\mu}$, where $m$ is the particle's mass, the four-velocity $u^{\mu}=\frac{\mathrm{d} x^{\mu}}{\mathrm{d} \tau}$, where $\tau$ is the proper time, $x^{\mu}$ are coordinates. Then the generalized momentum is equal to

$p_{\mu}=P_{\mu}-q A_{\mu}$,

$q$ is the particle's electric charge. Due to the symmetry of the metric, $P_{0}=-E$ and $P_{\phi}=L$ are conserved, where $E$ is the energy, and $L$ is the angular momentum.

We consider motion constrained within the equatorial plane, so $\theta=\frac{\pi}{2}$. Redefining the radial coordinate $r \rightarrow \rho$, we can always achieve that

$A=N^{2}$ 
within this plane. Then the equations of motion give

$\dot{t}=\frac{X}{N^{2} m}$,

$\dot{\phi}=\frac{\beta}{R}+\frac{\omega X}{m N^{2}}$,

$m^{2} \dot{\rho}^{2}+V=0$,

$X=E-\omega L$,

$\beta=\frac{\mathcal{L}}{R}-\frac{q B R}{2 m}$,

$V=m^{2} N^{2}\left(1+\beta^{2}\right)-X^{2}$.

A dot denotes differentiation with respect to the proper time $\tau$. As usual, we assume the forward in time condition $\dot{t}>0$, so $X \geq 0$. Hereafter, we use the notations

$\mathcal{L} \equiv \frac{L}{m}, \quad \mathcal{E}=\frac{E}{m}, \quad b=\frac{q B_{+} R_{+}}{2 m}$.

The subscripts "+", " 0 " denote quantities calculated on the horizon and ISCO, respectively.

In what follows, we will use the Taylor expansion of the quantity $\omega$ near the horizon. We denote $x=\rho-\rho_{+}$, where $\rho_{+}$is the horizon radius. Then

$\omega=\omega_{+}-a_{1} x+a_{2} x^{2}+\cdots$

\section{Equations determining ISCO}

By definition, ISCO is determined by the equations [5]

$V\left(\rho_{0}\right)=0$,

$\frac{\mathrm{d} V}{\mathrm{~d} \rho}\left(\rho_{0}\right)=0$,

$\frac{\mathrm{d}^{2} V}{\mathrm{~d} \rho^{2}}\left(\rho_{0}\right)=0$.

Equations (10) and (13) entail

$X\left(\rho_{0}\right)=m N\left(\rho_{0}\right) \sqrt{1+\beta^{2}\left(\rho_{0}\right)}$,

and Eqs. (14) and (15) turn into

$\frac{1}{m^{2}} \frac{\mathrm{d} V_{\text {eff }}}{\mathrm{d} \rho}=\frac{\mathrm{d}}{\mathrm{d} \rho}\left[N^{2}\left(1+\beta^{2}\right)\right]+2 \mathcal{L} \omega^{\prime} \sqrt{1+\beta^{2}} N=0$,

$$
\begin{aligned}
\frac{1}{m^{2}} \frac{\mathrm{d}^{2} V_{\text {eff }}}{\mathrm{d} \rho^{2}}= & \frac{\mathrm{d}^{2}}{\mathrm{~d} \rho^{2}}\left[N^{2}\left(1+\beta^{2}\right)\right]-2 \mathcal{L}^{2} \omega^{\prime} \sqrt{1+\beta^{2}} \omega^{\prime 2} \\
& +2 \mathcal{L} \omega^{\prime} \sqrt{1+\beta^{2}} \omega^{\prime \prime} N \sqrt{1+\beta^{2}}=0,
\end{aligned}
$$

where all quantities in (17) and (18) are to be taken at $\rho=$ $\rho_{0}$. A prime denotes the derivative with respect to $\rho$ (or, equivalently, $x$ ).
In general, it is impossible to find exact solutions of Eqs. (17) and (18). Therefore, in the next sections we analyze separately different particular situations, with main emphasis made on the near-horizon region. In doing so, we develop different versions of the perturbation theory that generalize the ones of [13]. The radius of ISCO, its energy, and angular momentum are represented as some series with respect to the corresponding small parameter, truncated at the leading or subleading terms, similarly to [13].

\section{Near-extremal black holes}

Let us consider a nonextremal black hole. In what follows, we are interested in the immediate vicinity of the horizon and use the Taylor series for corresponding quantities. Then near the horizon we have the expansion

$N^{2}=2 \kappa x+D x^{2}+C x^{3} \ldots$,

where $\kappa$ has the meaning of the surface gravity.

By definition, we call a black hole near-extremal if

$\kappa \ll D x_{0}$,

where $x_{0}=\rho_{0}-\rho_{+}$. Then for the lapse function we have an expansion near ISCO,

$N=x \sqrt{D}+\frac{\kappa}{\sqrt{D}}-\frac{\kappa^{2}}{2 D^{3 / 2} x}+\frac{C}{2 \sqrt{D}} x^{2}+\cdots$

Taking into account (18), after straightforward (but somewhat cumbersome) calculations, one can find that

$$
\begin{aligned}
& -\frac{1}{2} \frac{\mathrm{d} V_{\text {eff }}}{\mathrm{d} \rho}\left(\rho_{0}\right)=A_{2} x^{2}+A_{3} \frac{\kappa^{2}}{x}+\cdots, \\
& \mathcal{L} a_{1} \approx \sqrt{D P} \\
& P \equiv 1+\beta^{2} \\
& A_{2} \approx \frac{D}{2} \frac{\mathrm{d} P}{\mathrm{~d} x}+\frac{C P}{2}+\frac{a_{2}}{a_{1}} P D \\
& A_{3}=-\frac{P}{2 D}
\end{aligned}
$$

where $P$ and $\frac{\mathrm{d} P}{\mathrm{~d} x}$ are to be taken at $x=x_{0}$ or, with the same accuracy, at $x=0$ (i.e., on the horizon).

Then

$$
\begin{aligned}
& x_{0}^{3} \approx-\frac{A_{3}}{A_{2}} \kappa^{2}=H^{3} \kappa^{2}, \\
& H=\left(\frac{P_{0}}{2 D A_{2}}\right)^{1 / 3}=\frac{1}{\left[D\left(2 \frac{a_{2}}{a_{1}} D+C+\frac{D}{P} \frac{\mathrm{d} P}{\mathrm{~d} x}\right)\right]^{1 / 3}} .
\end{aligned}
$$


From (16) and (21) we have

$N_{0} \approx \sqrt{D} H \kappa^{2 / 3}$,

$X_{0} \approx m \sqrt{P_{+}} \sqrt{D} H \kappa^{2 / 3}$.

Using (23), (24), and (9) we derive an equation for the value of the angular momentum $L_{0}$ on ISCO:

$\frac{\mathcal{L}_{0}^{2}}{R_{+}^{2}}+2 b \frac{D}{d-D} \frac{\mathcal{L}_{0}}{R_{+}}-\frac{D\left(1+b^{2}\right)}{d-D}=0$,

where

$d \equiv R_{+}^{2} a_{1}^{2}$.

To have a well-defined limit $b=0$, we require $d-D>0$. We are interested in the positive root according to (23). Then

$\frac{\mathcal{L}_{0}(b)}{R_{+}}=-\frac{b D}{d-D}+\frac{\sqrt{D}}{d-D} \sqrt{d\left(1+b^{2}\right)-D}$,

and, in a given approximation,

$$
\begin{aligned}
& \beta_{0}= \frac{1}{d-D}\left[\sqrt{D} \sqrt{d\left(1+b^{2}\right)-D}-b d\right], \\
& P_{0}= \frac{d}{d-D}-2 \frac{b \sqrt{D} d}{(d-D)^{2}} \sqrt{d\left(1+b^{2}\right)-D} \\
&+\frac{b^{2} d(d+D)}{(d-D)^{2}}, \\
&\left(\frac{\mathrm{d} \beta}{\mathrm{d} x}\right)_{+}=-\frac{R_{+}^{\prime}}{R_{+}}\left[b+\frac{\mathcal{L}_{0}(b)}{R_{+}}\right]-\frac{B_{+}^{\prime}}{B_{+}} b, \\
& A_{2} \approx D \beta_{0}\left(\frac{\mathrm{d} \beta}{\mathrm{d} x}\right)_{+}+\frac{C P_{0}}{2}+\frac{a_{2}}{a_{1}} P_{0} D,
\end{aligned}
$$

where we neglected the difference between $\left(\frac{\mathrm{d} \beta}{\mathrm{d} x}\right)_{+}$and $\left(\frac{\mathrm{d} \beta}{\mathrm{d} x}\right)_{0}$. Equations (33)-(37) give the expression for $H$ after substitution into (28). To avoid cumbersome expressions, we leave it in the implicit form.

Now, two different limiting cases can be considered.

\subsection{Small magnetic field}

If $B=0$,

$$
\begin{aligned}
& \mathcal{L}_{0}(0)=\frac{\sqrt{D}}{\sqrt{a_{1}^{2}-\frac{D}{R_{+}^{2}}}}, \\
& \beta(0)=\frac{\sqrt{D}}{\sqrt{d-D}}, \\
& P_{0}(0) \approx \frac{d}{d-D}=\frac{R_{+}^{2} a_{1}^{2}}{R_{+}^{2} a_{1}^{2}-D},
\end{aligned}
$$

which agrees with Eq. (44) of Ref. [15]. It follows from (16) and (30) that

$\mathcal{E}(0) \approx \omega_{+} \frac{\sqrt{D}}{\sqrt{a_{1}^{2}-\frac{D}{R_{+}^{2}}}}$.

Let us consider a small but nonzero $b$. We can find from (33) that

$$
\mathcal{L}_{0}(b) \approx \mathcal{L}_{0}(0)-\mathcal{L}_{0}^{2}(0) \frac{b}{R_{+}}+O\left(b^{2}\right),
$$

$\mathcal{E}_{0}(b) \approx \omega_{0} \mathcal{L}_{0}+O\left(\kappa^{2 / 3}, b^{2}\right)$.

\subsection{Large magnetic field}

Let $b \gg 1$. Now, $P_{0} \sim b^{2}, A_{2} \sim b^{2}$. According to (27), there exists a finite $\lim _{B \rightarrow \infty} H=H_{\infty}$. In doing so, we find from (33), (9), and (30)

$$
\begin{aligned}
& \frac{\mathcal{L}_{0}}{R_{+}} \approx \frac{b \sqrt{D}}{\sqrt{d}+\sqrt{D}}=\frac{b \sqrt{D}}{a_{1} R_{+}+\sqrt{D}} \\
& \beta \approx-\frac{\sqrt{d} b}{\sqrt{d}+\sqrt{D}}=-\frac{R_{+} a_{1} b}{R_{+} a_{1}+\sqrt{D}} \\
& P_{0} \approx b^{2} \frac{d}{(\sqrt{d}+\sqrt{D})^{2}} \\
& X_{0} \approx m \sqrt{D} H_{\infty} \kappa^{2 / 3} \frac{b a_{1} R_{+}}{a_{1} R_{+}+\sqrt{D}} \\
& \mathcal{E} \approx b \sqrt{D}\left[\frac{\omega_{+} R_{+}}{a_{1} R_{+}+\sqrt{D}}+\sqrt{D} H_{\infty} \kappa^{2 / 3} \frac{a_{1} R_{+}}{a_{1} R_{+}+\sqrt{D}}\right]
\end{aligned}
$$

Thus according to (27), in general the radius of ISCO depends on the value of the magnetic field via the coefficient $H$. However, there is an exception. Let

$C=0, \quad R_{+}^{\prime}=0, \quad B^{\prime}=0$.

Then

$H^{3}=-\frac{A_{3}}{A_{2}}=\frac{a_{2}}{2 a_{1}} \frac{1}{D^{2}}$,

so the dependence on $b$ drops out from the quantity $H$ and, correspondingly, from the ISCO radius (27). One can check easily that the conditions (49) are satisfied for the nearextremal Kerr metric in the magnetic field. This agrees with Eq. (38) of [13] where the observation was made that in the main corrections of the order $\kappa^{2 / 3}$ the magnetic field does not show up. Thus this is the point where dirty black holes behave qualitatively differently from the Kerr metric in that the dependence of the ISCO radius on $b$ is much stronger than in the Kerr case.

It is instructive to evaluate the relation between $H(0)$ and $H(\infty)$ for vanishing and large magnetic fields, which results, 
according to (27), in different values of the corresponding radii $x_{0}$. The dependence on the magnetic field is due to the term $\frac{1}{P} \frac{\mathrm{d} P}{\mathrm{~d} x}$ in the denominator,

$$
\frac{H^{3}(0)}{H^{3}(\infty)}=\frac{2 \frac{a_{2}}{a_{1}} D+C+D w_{b=\infty}}{2 \frac{a_{2}}{a_{1}} D+C+D w_{b=0}}, w \equiv \frac{1}{P} \frac{\mathrm{d} P}{\mathrm{~d} x} .
$$

One can find that

$$
\begin{aligned}
& w_{b=0}=-\frac{2 R_{+}^{\prime}}{R_{+}} \frac{D}{d} \\
& w_{b=\infty}=-2\left\{\frac{R_{+}^{\prime}}{R_{+}}\left[\frac{2 \sqrt{D}+\sqrt{d}}{\sqrt{d}+\sqrt{D}}\right]+\frac{B_{+}^{\prime}}{B_{+}}\right\} \frac{(\sqrt{d}+\sqrt{D})}{\sqrt{d}} .
\end{aligned}
$$

Thus for $d \sim D \sim C, H(0) \sim H(\infty)$. However, in general they can differ significantly. Say, for $C=0=B_{+}^{\prime}$ and $d \ll D, d \ll D \frac{R_{+}^{\prime}}{R_{+}} \frac{a_{1}}{a_{2}}$, we have $\frac{H^{3}(0)}{H^{3}(\infty)} \approx \frac{2 d}{D} \ll 1$. As a result, the ISCO radius (27) also may vary over wide range.

\section{Slowly rotating black hole}

Now, we assume that $\kappa$ is not small, so the first term in (19) dominates. Here, we will consider different cases separately.

\subsection{Nonrotating black hole}

Here, we generalize the results known for the Schwarzschild black hole $[25,26]$ to a more general metric of a dirty static black hole. In Eqs. (14) and (15) we should put $a_{1}=0=a_{2}$. For a finite value of the magnetic field parameter $b$, ISCO lies at some finite distance from the horizon. However, now we will show that in the limit $b \rightarrow \infty$, the radius of ISCO tends to that of the horizon with $x_{0} \sim b^{-1}$.

We will show that this indeed happens, provided the term with $L$ in (9) is large and compensates the second one with $b$. Correspondingly, we write

$\mathcal{L}=\mathcal{L}_{0}+\mathcal{L}_{1}$,

where

$\frac{\mathcal{L}_{0}}{R_{+}}=b$.

For what follows, we introduce the quantity

$\alpha=\frac{\mathcal{L}_{1}}{R_{+}}$,

$\alpha=O(1)$. Then, near the horizon, where $x$ is small, we can use the Taylor expansion $\beta=\alpha-2 \frac{\beta_{0}}{R_{+}} x-x \alpha \frac{R_{+}^{\prime}}{R_{+}}+\frac{\beta_{2}}{R_{+}^{2}} b x^{2}+\cdots$,

$\beta_{2}=R_{+}^{\prime 2}-R_{+} R_{+}^{\prime \prime}-\frac{R_{+}^{\prime} R_{+} B_{+}^{\prime}}{B_{+}}-\frac{R_{+}^{2} B_{+}^{\prime \prime}}{2 B_{+}}$,

where

$\beta_{0}=b s$,

$s=R_{+}^{\prime}+\frac{1}{2} \frac{B_{+}^{\prime} R_{+}}{B_{+}}$.

Now, $\beta_{0} \gg 1$ but, by assumption, $\beta$ is finite.

In terms of the variable

$u=\frac{\beta_{0}}{R_{+}} x$,

it can be rewritten as

$\beta=\alpha-2 u+\frac{u}{\beta_{0}}\left(\frac{\beta_{2} u}{s}-\alpha c\right)+O\left(\beta_{0}^{-2}\right)$,

$c=R_{+}^{\prime}$.

It is clear from the above formulas that the expansion with respect to the coordinate $x$ is equivalent to the expansion with respect to inverse powers of the magnetic field $b^{-1}$, so for $b \gg 1$ this procedure is reasonable.

Then, after substitution of (62), we can represent (14) and (15) in the form of an expansion with respect to $\beta_{0}^{-1}$ :

$$
\begin{aligned}
& \frac{1}{m^{2}} \frac{\mathrm{d} V_{\text {eff }}}{\mathrm{d} \rho}=C_{0}+\frac{C_{1}}{\beta_{0}}+O\left(\beta_{0}^{-2}\right)=0, \\
& -\frac{1}{2 m^{2}} \frac{\mathrm{d}^{2} V_{\text {eff }}}{\mathrm{d} \rho^{2}}=-S_{1} \beta_{0}-S_{0}+O\left(\beta_{0}^{-1}\right)=0 .
\end{aligned}
$$

Here, the coefficients at leading powers are equal to

$C_{0}=2 \kappa\left(12 u^{2}-8 u \alpha+1+\alpha\right)$,

$S_{1}=16 \kappa(3 u-\alpha)$.

Then, in the main approximation, we have the equations $C_{0}=0$ and $S_{1}=0$ which give us

$u=\frac{1}{\sqrt{3}}, \quad \alpha=\sqrt{3}, \quad \beta=\frac{1}{\sqrt{3}}$.

To find the corrections $O\left(b^{-1}\right)$, we solve Eqs. (64) and (65) perturbatively. In doing so, it is sufficient to substitute these values into the further coefficients $C_{1}$ and $S_{0}$. The results are listed in the appendix.

In the particular case of the Schwarschild metric, $c=$ $\beta_{2}=s=1, D=-r_{+}^{-2}, \kappa=\left(2 R_{+}\right)^{-1}$, and $R_{+}=r_{+}$. Writing $r_{+}=2 M$, where $M$ is the black hole mass, we have from (54), (56), (61), (183), (185), and (187) 
$\frac{r_{0}-r_{+}}{M} \approx \frac{2}{\sqrt{3} b}-\frac{8}{3 b^{2}}$

$\frac{\mathcal{L}}{R_{+}} \approx b+\sqrt{3}-\frac{1}{3 b}$

$\mathcal{E}_{0} \approx \frac{2}{3^{3 / 4} \sqrt{b}}$.

Equations (69) and (71) agree with [12,13].

It is interesting that in terms of the variables $u, \frac{\mathcal{L}_{0}}{R_{+}}$, and $b$ the result (68) looks model-independent in the main approximation. This can be thought of as a manifestation of the universality of black hole physics near the horizon. The dependence on a model reveals itself in higher-order corrections.

\subsection{Extremely slow rotation}

Now, we consider rotation as the perturbation. Here, the angular velocity of rotation is the smallest parameter. Correspondingly, in Eqs. (18) and (15) we neglect the term $L^{2}$, since it contains $\omega^{\prime 2}$. More precisely, we assume

$\mathcal{L} a_{1}^{2} \ll a_{2} N$

so from (184) and (185) we have

$R_{+} b^{3 / 2} a_{1}^{2} \ll a_{2} \sqrt{\kappa R_{+}}$.

In the particular case of the slowly rotating Kerr metric, $\kappa \approx \frac{1}{2 R_{+}}, a_{1} \sim \frac{a}{M^{3}}=\frac{a^{*}}{M^{2}}, a_{2} \sim \frac{a}{M^{4}}=\frac{a^{*}}{M^{3}}$, where $a=$ $J / M, J$ is the angular momentum of a black hole, $a^{*}=\frac{a}{M}$. Then (72) reads

$a^{*} b^{3 / 2} \ll 1$

There are two kinds of corrections-due to the magnetic field and due to rotation. One can check that the presence of rotation leads to the appearance in the series (64) and (65) of half-integer inverse powers of $\beta_{0}$, in addition to integer ones. In the main approximation, we consider both kinds of corrections as additive contributions. Omitting details, we list the results:

$$
\begin{aligned}
& u \approx \frac{1}{\sqrt{3}}+\frac{1}{3 b s}\left(\frac{5}{6} \frac{\beta_{2}}{s}+\frac{1}{3} \frac{D R_{+}}{\kappa}-\frac{3 c}{2}\right) \\
&-\frac{\sqrt{2} \sqrt{R_{+}}}{3^{5 / 4} \sqrt{\kappa} \sqrt{s}} a_{1} R_{+} \sqrt{b} \\
& \frac{\mathcal{L}}{R_{+}} \approx b+\sqrt{3}-\sqrt{2} \frac{1}{3^{3 / 4} \sqrt{\kappa} \sqrt{s}} a_{1} R_{+}^{3 / 2} \sqrt{b}, \\
& N_{0} \approx \frac{\sqrt{2 \kappa R_{+}}}{3^{1 / 4} \sqrt{b s}} .
\end{aligned}
$$

It follows from (8) and (16) that

$$
\begin{aligned}
& X \approx \frac{2^{3 / 2} m}{3^{3 / 4}} \sqrt{\frac{\kappa R_{+}}{b s}}, \\
& \mathcal{E} \approx R_{+} \omega_{+} b+\frac{\sqrt{\kappa R_{+}}}{\sqrt{b} \sqrt{s}} \frac{2^{3 / 2}}{3^{3 / 4}} .
\end{aligned}
$$

For the slowly rotating Kerr metric, $R_{+} \approx 2 M$,

$\omega=\frac{R_{+} a}{r^{3}}+O\left(a^{2}\right)$.

In the main approximation the difference between the BoyerLindquist coordinate $r$ and the quasiglobal one $\rho$ has the same order $a^{2}$ and can be neglected. Then

$a_{1}=\frac{3 a^{*}}{2 R_{+}^{2}}$,

$u \approx \frac{1}{\sqrt{3}}+\frac{1}{\sqrt{3} b}-\frac{4}{3 b^{2}}-\frac{1}{3^{1 / 4}} \frac{a^{*}}{\sqrt{b}}$

$\mathcal{E} \approx \frac{1}{\sqrt{b}} \frac{2}{3^{3 / 4}}+\frac{a^{*}}{2} b$

$\frac{\mathcal{L}}{R_{+}} \approx b+\sqrt{3}-3^{3 / 4} a^{*} \sqrt{b}$.

They agree with the results of Sect. 3 B 2 of [13]. It is seen from (82)-(84) that the fractional corrections have the order $a^{*} b^{3 / 2}$ and are small in accordance with (74). In the more general case, the small parameter of the expansion corresponds to (73), so it is the quantity $\frac{\sqrt{R_{+}} b^{3 / 2} a_{1}^{2}}{a_{2} \sqrt{\kappa}}$.

\subsection{Modestly slow rotation}

Let now, instead of (72) and (73), the opposite inequalities hold:

$\mathcal{L} a_{1}^{2} \gg a_{2} N$

$R_{+} b^{3 / 2} a_{1}^{2} \gg a_{2} \sqrt{\kappa R_{+}}$,

or

$a^{*} b^{3 / 2} \gg 1$

in the Kerr case. Correspondingly, in what follows the small parameter of expansion is $\frac{a_{2} \sqrt{\kappa}}{\sqrt{R_{+}} b^{3 / 2} a_{1}^{2}}$, which reduces to $\left(a^{*} b^{3 / 2}\right)^{-1}$ in the Kerr case.

Additionally, we assume that

$b a_{1}^{* 2} \gg 1$.

It turns out (see the details in the appendix) that

$x_{0} \approx \frac{R_{+} \delta^{2}}{36} a_{1}^{* 2}$, 
$\omega_{0} \approx \omega_{+}-\frac{\delta^{2}}{36 R_{+}} a_{1}^{* 3}$,

$\frac{\mathcal{L}}{R_{+}}=b\left(1-\frac{\delta^{2}}{6} s a_{1}^{* 2}\right)$,

$N_{0} \approx \frac{1}{3 \sqrt{2}} \sqrt{\kappa R_{+}} a_{1}^{*} \delta$,

where $\delta=\frac{1}{s \sqrt{2 \kappa R_{+}}}$.

It follows from (190), (191), and (199) that

$\beta_{+}=\beta(0) \approx-\frac{1}{2} \beta_{0} a_{1}^{* 2}$,

$\beta\left(x_{0}\right) \approx-2 \frac{\delta^{2}}{9} b s a_{1}^{* 2}$.

$X_{0} \approx m \frac{1}{27} \sqrt{2 \kappa R_{+}} \delta^{3} b s a_{1}^{* 3}$,

$\mathcal{E}_{0} \approx \omega_{+} R_{+} b+v b a_{1}^{* 3}$,

$v=\frac{1}{27} \sqrt{2 \kappa R_{+}} s \delta^{3}-R_{+} s \delta^{2} \frac{\omega_{+}}{a_{1}^{*}}-\frac{\delta^{2}}{36}$.

\subsubsection{Kerr metric}

In the case of the slowly rotating Kerr black hole, Eq. (81) entails

$a_{1}^{*}=\frac{3}{2} a^{*}$

$\delta=1=s$,

$x_{0} \approx \frac{R_{+}}{36} a_{1}^{2 *}=\frac{R_{+}}{16} a^{* 2}$,

where we used (96).

One should compare this result to that in [13]. Now, $R_{+}=$ $2 M$, the horizon radius of the Kerr metric $r_{+} \approx 2 M\left(1-\frac{a^{* 2}}{4}\right)$. Equation (53) of [13] gives us

$r_{0} \approx 2 M\left(1-\frac{3 a^{* 2}}{16}\right)$

whence $x_{0}=r_{0}-r_{+} \approx \frac{R_{+}}{16} a^{* 2}$, which coincides with (99). It is seen from (91) and (98) that the angular momentum takes the value

$\frac{\mathcal{L}}{R_{+}} \approx b\left(1-\frac{3}{8} a^{* 2}\right)$

which coincides with Eq. (55) of [13]. Also, one finds that

$X_{0} \approx \frac{m}{8} b a^{* 3}$

In Eq. (90) one should take into account that $\omega_{+}$depends on $r_{+}$, which itself can be expressed in terms of $a^{*}$ and $M$.
Collecting all terms, one obtains from (96)

$\mathcal{E}_{0} \approx \frac{a^{*} b}{2}-\frac{b a^{* 3}}{32}$

which agrees with Eq. (54) of [13].

\section{ISCO for rotating nonextremal black holes in a strong magnetic field}

In the previous section we saw that in the limit $b \rightarrow \infty$ the ISCO radius does not coincide with that of the horizon, which generalizes the corresponding observation made in Sect. III B 3 of [13]. Now, we will see that this is a general result which is valid for an arbitrary degree of rotation and finite $\kappa$ (so, for generic nonextremal black holes). It is worth noting that for $b=0$ it was noticed that the near-horizon ISCO are absent $[15,27]$. However, for $b \gg 1$ the corresponding reasonings do not apply, so we must consider this issue anew.

We have to analyze Eqs. (17) and (18) in which (16) is taken into account.

Neglecting higher-order corrections, we can rewrite them in the form

$$
\begin{aligned}
& (2 \kappa+2 D x)\left(1+\beta^{2}\right)+\left(2 \kappa x+D x^{2}\right) \frac{\mathrm{d} \beta^{2}}{\mathrm{~d} x} \\
& -\frac{2 L \sqrt{1+\beta^{2}}}{m} N\left(a_{1}-2 a_{2} x\right)=0, \\
& \frac{2 L^{2}}{m^{2}}\left(a_{1}-2 a_{2} x\right)^{2}-2 a_{2} N \sqrt{1+\beta^{2}} \frac{L}{m}-W=0, \\
& W=(2 D+6 C x)\left(1+\beta^{2}\right)+2 \frac{\mathrm{d} \beta^{2}}{\mathrm{~d} x}(2 \kappa+2 D x) \\
& \quad+\left(2 \kappa x+D x^{2}\right) \frac{\mathrm{d}^{2} \beta^{2}}{\mathrm{~d} x^{2}} .
\end{aligned}
$$

(1) Let us suppose that $\beta$ is finite or, at least, $\beta \ll b$. Then it follows from (57), (9), and (55) that $\frac{\mathrm{d} \beta}{\mathrm{d} x} \sim b$ and $L \sim b$. Also, $x \sim b^{-1}$ according to (61), $N \sim \sqrt{x} \sim b^{-1 / 2}$. However, it is impossible to compensate the term with $L^{2}$ in (105) having the order $b^{2}$.

(2) Let $\beta \sim L \sim b$. Then in (104) the first term has the order $b^{2}$ and cannot be compensated.

(3) Let $\beta \gg b$. Then Eq. (9) gives $L \sim \beta$. Again, the first term in (104) cannot be compensated.

Thus we see that, indeed, in the limit $b \rightarrow \infty$ the assumption about $x \rightarrow 0$ leads to contradictions, so the ISCO radius does not approach the horizon.

\section{Extremal nonrotating black hole}

Up to now, we considered the case of a nonextremal black hole, so the surface gravity $\kappa$ was arbitrary or a small quantity 
but it was nonzero anyway. Let us discuss now the case of the extremal black hole, so $\kappa=0$ exactly. We pose the question: is it possible to get an ISCO such that for $b \rightarrow \infty$ the ISCO radius tends to that of the horizon? Now, we will see that this is indeed possible for a nonrotating black hole $(\omega=$ $0)$. We assume that the electric charge that can affect the metric is negligible. The extremal horizon appears due to the properties of the matter that surrounds the horizon, which is possible even in the absence of the electric charge, provided the equation of state obeys some special conditions [23,24].

For ISCO close to the horizon we can use the expansion

$N^{2}=D x^{2}+\cdots$,

in which we drop the terms of the order $x^{3}$ and higher. Now we show that the case under discussion does exist with a finite quantity $\beta$. We can use now (62) in which only the first term is retained, so

$\beta \approx \alpha-2 u$

where $u$ is given by Eq. (61). Then Eq. (10) reads

$V \approx m^{2} \frac{D R_{+}^{2}}{b^{2} s^{2}} f(u)-E^{2}$,

where

$f(u)=u^{2}\left(1+\alpha^{2}-4 u \alpha+4 u^{2}\right)$.

Equations (14) and (15) reduce to

$\frac{\mathrm{d} f}{\mathrm{~d} u}\left(u_{0}\right)=0$,

$\frac{\mathrm{d}^{2} f}{\mathrm{~d} u^{2}}\left(u_{0}\right)=0$.

They have the solution

$u_{0}=\frac{3}{2^{3 / 2}}, \quad \alpha=\frac{4}{\sqrt{2}}=2 \sqrt{2}$,

whence

$\beta \approx \frac{1}{\sqrt{2}}$

Correspondingly, Eqs. (107) and (13) give us

$N\left(x_{0}\right) \approx \frac{3}{2^{3 / 2}} \sqrt{D} \frac{R_{+}}{b s}$,

$\frac{X_{0}}{m}=\mathcal{E}_{0} \approx \frac{3^{3 / 2}}{4} \sqrt{D} \frac{R_{+}}{b s}$.

We can also find the angular momentum on ISCO

$\frac{\mathcal{L}_{0}}{R_{+}} \approx b+\frac{1}{2} \sqrt{2}$

Thus for big $b$ there is ISCO outside the horizon that tends to it in the limit $b \rightarrow \infty$, so that the quantity $x_{0} \rightarrow 0$.

\section{Extremal rotating black hole}

Now, we consider the same question but now for rotating black holes: is it possible to have ISCO in the near-horizon region (as closely as we like) for the extremal $\mathrm{BH}$, when $\kappa=0$ ? Mathematically, it would mean that

$\lim _{b \rightarrow \infty} x_{0}=0$.

Then Eqs. (17) and (21) with $\kappa=0$ give for small $x$

$$
\begin{aligned}
& x_{0} D\left[\left(1+\beta^{2}\right)-\frac{L a_{1} \sqrt{1+\beta^{2}}}{m \sqrt{D}}\right] \\
& +\frac{x_{0}^{2}}{2}\left\{C\left[3\left(1+\beta^{2}\right)-\frac{L a_{1} \sqrt{1+\beta^{2}}}{m \sqrt{D}}\right]+D\left(\beta^{2}\right)^{\prime}\right\}=0 .
\end{aligned}
$$

Equation (18) with terms of the order $x_{0}^{2}$ and higher neglected, gives rise to

$$
\begin{aligned}
& D\left(1+\beta^{2}\right)-\mathcal{L}^{2} a_{1}^{2}+x_{0}\left[2 D\left(\beta^{2}\right)^{\prime}+2 a_{2} \mathcal{L}^{2} \sqrt{D} \sqrt{1+\beta^{2}\left(x_{0}\right)}\right. \\
& \left.+2 \mathcal{L}^{2} a_{1} a_{2}+3 C\left(1+\beta^{2}\right)\right]=0 .
\end{aligned}
$$

Then the main terms in (119) and (120) entail

$\mathcal{L} a_{1}=\sqrt{D} \sqrt{1+\beta^{2}}$.

For $b \gg 1$, assuming for definiteness that $d>D(d$ is defined according to (32)), one finds from (9) and (121) that

$\beta_{+} \approx-b \frac{\sqrt{d}}{\sqrt{d}+\sqrt{D}}$

$\frac{\mathcal{L}}{R_{+}}=b \frac{\sqrt{D}}{\sqrt{d}+\sqrt{D}}$,

$\frac{L^{2}}{m^{2}} a_{1}^{2}=D+D\left(\frac{L^{2}}{m^{2} R_{+}^{2}}-2 \frac{L}{m R_{+}} b+b^{2}\right)$.

The terms $x_{0}^{2}$ in (119) and $x_{0}$ in (120) give us, with (121) taken into account

$\left(1+\beta^{2}\right) C+D\left(\beta^{2}\right)^{\prime}=0$,

$\left(\beta^{2}\right)^{\prime}+2 \frac{a_{2}}{a_{1}}\left(1+\beta^{2}\right)+\frac{3 C}{2 D}\left(1+\beta^{2}\right)=0$,

whence

$C=-4 D \frac{a_{2}}{a_{1}}$.

The system is overdetermined; Eq. (127) cannot be satisfied in general. In principle, one can consider (127) as a restriction on the black hole parameters. This is similar to the situation for the extremal Kerr-Newman metric $(b=0)$, where ISCO near the horizon exists only for the selected value of the angular momentum, approximately equal to 
$\frac{a}{M} \approx \frac{1}{\sqrt{2}}[8,9]$. However, we will not discuss such exceptional cases further. Generically, the answer to our question is negative, so the ISCO radius does not approach the horizon in the limit $b \rightarrow \infty$.

\section{Particle collisions: general formulas}

Let two particles collide. We label their characteristics by indices 1 and 2. Then, in the point of collision, one can define the energy in the center of mass (CM) frame as

$E_{\mathrm{c} . \mathrm{m} .}^{2}=-p_{\mu} p^{\mu}=m_{1}^{2}+m_{2}^{2}+2 m_{1} m_{2} \gamma$.

Here,

$p^{\mu}=m_{1} u_{1}^{\mu}+m_{2} u_{2}^{\mu}$

is the total momentum, and

$\gamma=-u_{1 \mu} u_{2}^{\mu}$

is the Lorentz factor of their relative motion.

For motion in the equatorial plane in the external magnetic field (2), one finds from the equations of motion (6) and (7) that

$\gamma=\frac{X_{1} X_{2}-\varepsilon_{1} \varepsilon_{2} \sqrt{V_{1} V_{2}}}{m_{1} m_{2} N^{2}}-\beta_{1} \beta_{2}$.

Here, $\varepsilon=+1$, if the particle moves away from the horizon, and $\varepsilon=-1$ if it moves toward it.

Now, there are two scenarios relevant in our context. We call them the $\mathrm{O}$-scenario and $\mathrm{H}$-scenario according to the terminology of [15]. Correspondingly, we will use the superscripts "O" and " $\mathrm{H}$ ".

\subsection{O-scenario}

Particle 1 moves on ISCO. As $V_{1}\left(\rho_{0}\right)=0$ on ISCO, the formula simplifies to

$\left(E_{\mathrm{c} . \mathrm{m} .}^{O}\right)^{2}=m_{1}^{2}+m_{2}^{2}+2\left(\frac{X_{1} X_{2}}{N^{2}}-m_{1} m_{2} \beta_{1} \beta_{2}\right)$.

As we are interested in the possibility to get $\gamma$ as large as one likes, we will consider the case when the ISCO is close to the horizon, so $N$ is small. In doing so, we will assume that $\left(X_{2}\right) \neq 0$, so particle 2 is usual according to the terminology of [15]. We also must take into account Eq. (16), whence

$\left(E_{\text {c.m. }}^{O}\right)^{2}=m_{1}^{2}+m_{2}^{2}+2\left(m_{1} \frac{X_{2} \sqrt{1+\beta_{1}^{2}}}{N}-m_{1} m_{2} \beta_{1} \beta_{2}\right)$.
For ISCO close to the horizon, the first term dominates and we have

$\left(E_{\mathrm{c} . \mathrm{m} .}^{O}\right)^{2} \approx 2 m_{1} \frac{\left(X_{2}\right)_{0}\left(\sqrt{1+\beta_{1}^{2}}\right)_{0}}{N_{0}}$.

\subsection{H-scenario}

Now, particle 1 leaves ISCO (say, due to additional collision) with the corresponding energy $E=E\left(x_{0}\right)$ and angular momentum $L=L\left(x_{0}\right)$, which corresponds just to ISCO. This particle moves toward the horizon where it collides with particle 2.

Mathematically, it means that we should take the horizon limit $N \rightarrow 0$ first in Eq. (131). We assume that both particles move toward the horizon, so $\varepsilon_{1} \varepsilon_{2}=+1$. Then

$$
\begin{aligned}
\left(E_{\mathrm{c} . \mathrm{m} .}^{H}\right)^{2}= & m_{1}^{2}+m_{2}^{2}+m_{1}^{2}\left(1+\beta_{1}^{2}\right) \frac{X_{2}}{X_{1}}+m_{2}^{2}\left(1+\beta_{2}^{2}\right) \frac{X_{1}}{X_{2}} \\
& -2 m_{1} m_{2} \beta_{1} \beta_{2},
\end{aligned}
$$

where all quantities are to be calculated on the horizon.

For small $X_{1}$, when

$X_{1} \ll X_{2} \frac{m_{1}}{m_{2}} \sqrt{\frac{1+\beta_{1}^{2}}{1+\beta_{2}^{2}}}$,

we see from (135) that

$\left(E_{\mathrm{c} . \mathrm{m} .}^{H}\right)^{2} \approx m_{1}^{2}\left(1+\beta_{1}^{2}\right)_{+} \frac{\left(X_{2}\right)_{+}}{\left(X_{1}\right)_{+}}$.

Now,

$X_{1}=E_{0}-\omega_{+} L_{0}=X_{0}+\left(\omega_{0}-\omega_{+}\right) L_{0}$,

where $X_{0}=E_{0}-\omega_{0} L$ corresponds to ISCO. With (12) and (16) taken into account, in the main approximation

$X_{1} \approx m_{1} N_{0} \sqrt{1+\beta_{1}^{2}\left(x_{0}\right)}-a_{1} x_{0} L_{0}$.

Now, we apply these formulas to the different cases considered above.

\section{Kinematics of motion on ISCO}

It is instructive to recall that the general explanation of high $E_{\text {c.m. }}$ consists in the simple fact that a rapid usual particle, having a velocity close to speed of light, hits the slow particle that has parameters approximately equal to the critical values. This was explained in detail in [16] for the standard BSW effect (without considering collision near ISCO). Does this explanation retain its validity in the present case? One particle that participates in a collision is usual, so it would cross the horizon with a velocity approaching the speed of light in an appropriate stationary frame (see below). We consider the near-horizon ISCO, so the velocity of a usual particle is close 
to the speed of light. Now, we must check what happens to the velocity of a particle on ISCO.

To describe the kinematic properties, it is convenient to introduce the tetrads that in the local tangent space enable us to use formulas similar to those of special relativity. A natural and simple choice is the tetrad of a so-called zeroangular observer (ZAMO) [5]. It reads

$h_{(0) \mu}=-N(1,0,0,0)$,

$h_{(1) \mu}=N^{-1}(0,1,0,0)$,

$h_{(2) \mu}=\sqrt{g_{\theta}}(0,0,1,1)$,

$h_{(3) \mu}=R(-\omega, 0,0,1)$.

Here, $x^{0}=t, x^{1}=r, x^{2}=\theta, x^{3}=\phi$. It is also convenient to define the local three-velocity [5] according to

$v^{(a)}=v_{(a)}=\frac{u^{\mu} h_{\mu(a)}}{-u^{\mu} h_{\mu(0)}}$,

$a=1,2,3$.

From the equations of motion (13)-(22) and the formulas for the tetrad components, we obtain

$-u^{\mu} h_{\mu(0)}=\frac{X}{m N}$,

$u^{\mu} h_{\mu(3)}=\beta$,

$v^{(3)}=\frac{m \beta N}{X}$,

$v^{(1)}=\sqrt{1-\frac{m^{2} N^{2}}{X^{2}}\left(1+\beta^{2}\right)}$,

the component $v^{(2)}=0$ for equatorial motion.

Then, introducing also the absolute value of the velocity $v$ according to

$v^{2}=\left[v^{(1)}\right]^{2}+\left[v^{(3)}\right]^{2}$,

one can find that

$X=m \gamma_{0} N, \quad \gamma_{0}=\frac{1}{\sqrt{1-v^{2}}}$.

Equation (150) was derived in [16] for the case when the magnetic field is absent. We see that its general form does not depend on the presence of such a field.

For a circle orbit, Eq. (16) should hold. Comparing it with (150), we find that

$\gamma_{0}=\sqrt{1+\beta^{2}}$,

which has the same form as for the static case [12]. Now we can consider different cases depending on the value of the magnetic field and the kind of black hole.

\subsection{Near-extremal black holes}

For small $b$, Eq. (39) shows that $\beta$ is finite, so is the quantity $\gamma_{0}$. Therefore, $v<1$. For the Kerr metric, in the main approximation, $\beta=\frac{L_{0}}{m R_{+}}=\frac{1}{\sqrt{3}}$ on ISCO (see Eq. 4.7 of [14] and Eq. 77 of [15], $R_{+}=2 M$ ), so $\gamma_{0}=\frac{2}{\sqrt{3}}, v=\frac{1}{2}$, which is a well-known result (see discussion after Eq. 3.12 b in [5]).

For large $b$, accoding to (59), the quantity $\beta$ is proportional to $b$, and grows, $v \rightarrow 1$. However, this case is not very interesting since the individual energy (48) diverges itself.

10.2 Nonrotating or slowly rotating nonextremal black holes

According to Eq. (68), $\beta \approx \frac{1}{\sqrt{3}}$. Slow rotation adds only small corrections to this value. Thus, rather unexpectedly, we again see that on ISCO

$v \approx \frac{1}{2}$.

This value coincides for the near-extremal Kerr without a magnetic field and a nonrotating or slowly rotating dirty black hole in the strong magnetic field.

10.3 Modestly rotating nonextremal black hole

It follows from (93) and (88) that $|\beta| \gg 1$. However, as the energy of a particle on ISCO (96) tends to inifnity, this case is also not so interesting.

To summarize, in all cases of interest (when an individual energy is finite), $\beta$ remains finite even in the strong magnetic field. Correspondingly, $v<1$ on ISCO and the previous explanation of the high $E_{\text {c.m. }}$ [16] applies. For less interesting cases, when an individual energy diverges, we have collision between two rapid particles but their velocities are not parallel and this also gives rise to high $\gamma_{0}$ (see Eq. 20 in [16]).

\section{Center-of-mass energy of collision}

\subsection{Near-extremal black hole}

\subsubsection{O-scenario}

Using (134), (21) and (29) one obtains

$\left(E_{\mathrm{c} . \mathrm{m} .}^{O}\right)^{2} \approx 2 m_{1} \frac{\left(X_{2}\right)+\sqrt{1+\beta_{1}^{2}}}{\sqrt{D} H \kappa^{2 / 3}}$.

In a strong magnetic field, with $b \gg 1$, using the expression (45) for $\beta$, we obtain 
$\left(E_{\mathrm{c} . \mathrm{m} .}^{O}\right)^{2} \approx \frac{2 m_{1}\left(X_{2}\right)_{+}}{\sqrt{D} H \kappa^{2 / 3}} \frac{R_{+} a_{1} b}{R_{+} a_{1}+\sqrt{D}}$.

In the near-extremal Kerr case, $D=M^{-2}, R_{+}=2 M$, $\kappa \approx \frac{1}{2} \sqrt{1-a^{* 2}}, H=M^{-5 / 3}, a_{1}=M^{-2}$. As a result,

$\left(E_{\mathrm{c} . \mathrm{m} .}^{O}\right)^{2} \approx \frac{2^{8 / 3} m_{1}\left(X_{2}\right)_{+} b}{3\left(1-a^{* 2}\right)^{1 / 3}}$,

which coincides with Eq. (61) of [13], in which the limit $b \rightarrow \infty$ should be taken.

\subsubsection{H-scenario}

Now, due to (121), Eq. (139) gives $X_{1}=0$. It means that in the expansion (21) we must retain the first correction in the expression for $N$, when it is substituted into (139). As a result, we have

$X_{1} \approx \frac{m_{1} \kappa \sqrt{1+\beta_{1}^{2}\left(x_{0}\right)}}{\sqrt{D}}$.

There are also terms of the order $x_{0}^{2} \sim \kappa^{4 / 3}$ but they are negligible as compared to $\kappa$. Correspondingly, Eq. (135) gives

$\left(E_{\mathrm{c} . \mathrm{m} .}^{H}\right)^{2} \approx m_{1} \sqrt{D} \sqrt{1+\beta_{1}^{2}}\left(X_{2}\right)_{+} \kappa^{-1}$.

In a strong magnetic field, with $b \gg 1$, using (45) again we obtain

$\left(E_{\mathrm{c} . \mathrm{m} .}^{H}\right)^{2} \approx m_{1} \sqrt{D} \frac{R_{+} a_{1} b}{R_{+} a_{1}+\sqrt{D}}\left(X_{2}\right)_{+} \kappa^{-1}$.

Thus in both versions, for $b \gg 1$ the effect is enhanced due to the factor $b$. For $b=0$ we return to [15].

In the Kerr case,

$\left(E_{\mathrm{c} . \mathrm{m} .}^{H}\right)^{2} \approx \frac{4}{3} m_{1} \frac{\left(X_{2}\right)_{+} b}{\left(1-a^{* 2}\right)^{1 / 2}}$,

which corresponds to Eq. (59) of [13], in which $b \gg 1$.

11.2 Extremely slowly rotating or nonrotating black hole

\subsubsection{O-scenario}

Now, Eqs. (184) and (134) give us

$\left(E_{\mathrm{c} . \mathrm{m} .}^{O}\right)^{2} \approx 3^{-1 / 4} \frac{4 m_{1}\left(X_{2}\right)_{+}}{\sqrt{2 \kappa R_{+}}} \sqrt{b s}$.

In the Schwarschild case, $2 \kappa R_{+}=1=s$,

$\left(E_{\mathrm{c} . \mathrm{m} .}^{O}\right)^{2} \approx \frac{4 m_{1}\left(X_{2}\right)_{+}}{3^{1 / 4}} \sqrt{b}$,

which coincides with Eq. (63) of [13].

\subsubsection{H-scenario}

Using (78) and neglecting in (139) the second term (rotational part), we get

$\left(E_{\mathrm{c} . \mathrm{m} .}^{H}\right)^{2} \approx \frac{2}{3^{3 / 4}} \frac{m_{1}\left(X_{2}\right)_{+}}{\sqrt{2 \kappa R_{+}}} \sqrt{b s}$.

11.3 Modestly rotating black holes in strong magnetic field

\subsubsection{O-scenario}

With $\beta_{1} \gg 1$, it follows from (92), (94), and (134) that

$\left(E_{\mathrm{c} . \mathrm{m} .}^{O}\right)^{2} \approx \frac{4}{3} \sqrt{2} \frac{m_{1}}{\sqrt{\kappa R_{+}}} \delta b s\left(X_{2}\right)_{+} a_{1}^{*}$.

In the Kerr case, taking into account (98), we obtain

$\left(E_{\mathrm{c} . \mathrm{m} .}^{O}\right)^{2} \approx 4 m_{1}\left(X_{2}\right)_{+} a^{*} b$,

which agrees with Eq. (67) of [13].

\subsubsection{H-scenario}

In a similar manner, one can obtain from (137), (139), and (95) that $\left(E_{\mathrm{c} . \mathrm{m} .}^{H}\right)^{2} \sim b$ with a somewhat cumbersome coefficient, which we omit here.

Both these scenarios are less interesting, since according to (96), the individual energy $\mathcal{E}_{0} \sim b$ diverges itself in the limit $b \rightarrow \infty$.

\subsection{Extremal nonrotating black holes}

\subsubsection{O-scenario}

Using (115), (114) we find from (134) that

$\left(E_{\mathrm{c} . \mathrm{m} .}^{O}\right)^{2} \approx \frac{4 m_{1}\left(X_{2}\right)_{+} b s}{\sqrt{3 D} R_{+}}$.

\subsubsection{H-scenario}

Now, it follows from (116), (114), and (137) that

$\left(E_{\mathrm{c} . \mathrm{m} .}^{H}\right)^{2} \approx 2 m_{1} \frac{\left(X_{2}\right)_{+} b s}{\sqrt{3 D} R_{+}} ;$

$\frac{b\left(1+\xi^{2}\right)}{\Lambda_{+}^{3}}$.

\section{Backreaction of magnetic field: Ernst static black hole}

Now, we illustrate the obtained results using the metric of a static magnetized black hole [28] that can be considered as the generalization of the Schwarzschild solution. This will 
also allow us to elucidate the role of backreaction due to the magnetic field on the behavior of $E_{\mathrm{c} . \mathrm{m}}$., which bounds the BSW effect. The metric reads

$$
\begin{aligned}
\mathrm{d} s^{2}= & \Lambda^{2}\left[-\left(1-\frac{r_{+}}{r}\right) \mathrm{d} t^{2}+\frac{\mathrm{d} r^{2}}{1-\frac{r}{r}}+r^{2} \mathrm{~d} \theta^{2}\right] \\
& +\frac{r^{2} \sin ^{2} \theta}{\Lambda^{2}} \mathrm{~d} \phi^{2}, \Lambda^{2}=1+\frac{B^{2} r^{2}}{4} \sin ^{2} \theta \\
A^{\phi}= & \frac{\tilde{B}}{2}, \tilde{B}=B \Lambda,
\end{aligned}
$$

$r_{+}=2 M$ is the horizon radius, $B$ is a constant parameter. It follows from (11) (with $B$ replaced with $\tilde{B}$ ) and (167) that

$\beta=\frac{\mathcal{L}}{R}-b \frac{r}{2 M}, \quad b=\frac{q B M}{m}$.

Many important details of the particle's motion in this background can be found in Ref. [29].

Calculating the corresponding coefficients according to (58)-(60) and substituting them into (182)-(187), we obtain

$$
\begin{aligned}
& \frac{\left(r-r_{+}\right)}{r_{+}}=\frac{(1+\xi)}{\left(1+\xi^{2}\right) b} \\
& \quad \times\left[\frac{1}{\sqrt{3}}+\frac{-8+18 \xi-3 \xi^{2}-2 \xi^{3}-\xi^{4}}{18 b\left(1+\xi^{2}\right)^{2}}\right] \\
& \xi=B^{2} M^{2} \\
& \frac{\mathcal{L}}{2 M}=\frac{1}{(1+\xi)}\left[b+\sqrt{3}+\frac{-1+3 \xi-\xi^{3}-\xi^{4}}{3 b\left(1+\xi^{2}\right)^{2}}\right]+O\left(b^{-2}\right) \\
& \mathcal{E}_{0} \approx \frac{2(1+\xi)^{3 / 2}}{3^{3 / 4} \sqrt{b}} \frac{1}{\sqrt{1+\xi^{2}}} .
\end{aligned}
$$

It follows from (186) that

$\beta \approx \frac{1}{\sqrt{3}}+\frac{(\xi-1)\left(-\xi^{3}-\xi^{2}-2 \xi+2\right)}{3 b\left(1+\xi^{2}\right)^{2}}$

For the energy of collision we have from (160) and (162)

$\left(E_{\mathrm{c} . \mathrm{m} .}^{O}\right)^{2} \approx 3^{-1 / 4} 4 m_{1}\left(X_{2}\right)_{+} z$

$\left(E_{\mathrm{c} . \mathrm{m} .}^{H}\right)^{2} \approx \frac{2}{3^{3 / 4}} m_{1}\left(X_{2}\right)_{+} z$

where

$z=\sqrt{\frac{b\left(1+\xi^{2}\right)}{(1+\xi)^{3}}}$.

When $\xi \ll 1$, there is agreement with the results for the Schwarzschild metric [12,13] since Eq. (170) turns into (69) and (172) turns into (70). It is interesting that for any $\xi$, the velocity of a particle on ISCO is equal to $1 / 2$ like this happens for $\xi \ll 1$. The approach under discussion works well also for $\xi \gg 1$, provided that the ISCO lies close to the horizon to ensure large $E_{\text {c.m. }}$, i,e, $N^{2} \ll 1$.According to (167) and (170), this requires

$b \gg \xi$

or, equivalently,

$\frac{q}{m} \gg B M \gg 1$.

Otherwise, both energies (175) and (176) contain the factor $\sqrt{\frac{b}{\xi}} \sim \sqrt{\frac{q}{m B M}}$, which bounds $E_{\text {c.m. }}$, which begins to decrease when $B$ increases. One should also bear in mind that it is impossible to take the limit $\xi \rightarrow \infty$ literally since the geometry becomes singular. In particular, the component of the curvature tensor $R_{\theta \phi}^{\theta \phi}$ grows like $\xi^{2}$. The maximum possible $E_{\text {c.m. }}$ is achieved when $\xi \sim 1$, then $z \sim \sqrt{\frac{q}{m}}$.

The example with the Ernst metric shows that strong backreaction of the magnetic field on the geometry may restrict the growth of $E_{\text {c.m. }}$. to such extent that even in spite of large $b$, the effect disappears because of the factor $\xi$ that enters the metric. It is of interest to consider the exact rotating magnetized black hole [30] that generalizes the Kerr metric but this problem certainly needs separate treatment.

\section{Summary and conclusion}

We obtained characteristics of ISCO and the energy in the $\mathrm{CM}$ frame in two different situations. For the near-extremal case, we considered the BSW effect. Previous results applied to the weakly magnetized Kerr metric or dirty black holes without the magnetic field. Now, we took into account both factors, so generalized the previous results for the case when both matter and magnetic field are present. In doing so, there is qualitative difference between dirty rotating black holes and the Kerr one. Namely, the radius of ISCO depends on the magnetic field strength $b$ already in the main approximation with respect to small surface gravity $\kappa$ in contrast to the case of the vacuum metric [13], where this dependence reveals itself in the small corrections only.

For extremal black holes, we showed that, due to the strong magnetic field, there exists the near-horizon ISCO that does not have a counterpart in the absence of this field. Correspondingly, we described the effect of high-energy collisions near these ISCO.

We demonstrated that rotation destroys near-horizon ISCO both for the nonextremal and extremal horizons, so $\lim _{b \rightarrow \infty} r_{0}(b) \neq r_{+}$. However, if the parameter responsible for rotation is small, $E_{\mathrm{c} . \mathrm{m}}$ is large in this limit.

For slowly rotating black holes we analyzed two different regimes of rotation thus having generalized previous results on the Kerr metric [13]. The parameters of expansion used in calculations and the results agree with the Kerr case. In 
particuar, for modestly slow rotation the individal energy of the particle on ISCO is unbound.

In the main approximation, the expressions for the ISCO radius and angular momentum in dimensionless variables are model-independent, so here one can see universality of black hole physics.

We also found the three-velocity of a particle on ISCO in the ZAMO frame. It turned out that for slowly rotating dirty black holes in the magnetic field it coincides with the value typical of the Kerr metric without a magnetic field, $v \approx \frac{1}{2}$. Correspondingly, the previous explanation of the high $E_{\mathrm{c} . \mathrm{m}}$. as the result of collisions of very fast and slow particles [16] retains its validity in the scenarios under discussion as well.

In previous studies of the BSW effect in the magnetic field [12,13,21], some fixed background was chosen. In this sense, the magnetic field was supposed to be weak in that it did not affect the metric significantly (although it influenced strongly motion of charged particles). Meanwhile, the most part of the formulas obtained in the present work applies to generic background and only asymptotic behavior of the metric near the horizon was used. Therefore, they apply to the backgrounds in which the magnetic field enters the metric itself, with reservation that the surface gravity $\kappa=\kappa(b)$, etc. In particular, we considered the static magnetized Ernst black hole and showed that strong backreaction of the magnetic field on the geometry bounds the growth of $E_{\text {c.m. }}$.

Thus we embedded previous scenarios of high-energy collisions in the magnetic field near ISCO in the vicinity of black holes $[12,13]$ and took into account the influence of the magnetic field on the metric.

Throughout the paper, it was assumed that the effect of the electric charge on the metric is negligible. It is of interest to extend the approach of the present work to the case of charged black holes.

Acknowledgments This work was funded by the subsidy allocated to Kazan Federal University for the state assignment in the sphere of scientific activities.

Open Access This article is distributed under the terms of the Creative Commons Attribution 4.0 International License (http://creativecomm ons.org/licenses/by/4.0/), which permits unrestricted use, distribution, and reproduction in any medium, provided you give appropriate credit to the original author(s) and the source, provide a link to the Creative Commons license, and indicate if changes were made.

Funded by SCOAP ${ }^{3}$.

\section{Appendix}

Here, we list some rather cumbersome formulas which are excluded from the main text.

A. Nonrotating black holes

$C_{1}=4 \kappa \frac{\beta_{2}}{3 \sqrt{3} s}+\frac{4}{3 \sqrt{3}} D R_{+}$,
$S_{0} \approx 8 \frac{\kappa}{R_{+}}\left(-\frac{\beta_{2}}{s}+3 c\right)$

The results with the leading term and subleading corrections read

$u \approx \frac{1}{\sqrt{3}}+\varepsilon_{1}, \quad \varepsilon_{1}=\frac{1}{3 b s}\left(\frac{5}{6} \frac{\beta_{2}}{s}+\frac{1}{3} \frac{D R_{+}}{\kappa}-\frac{3 c}{2}\right)$,

$\alpha \approx \sqrt{3}+\delta_{1}, \quad \delta_{1}=\frac{1}{3 b s}\left(\frac{\beta_{2}}{s}+\frac{D R_{+}}{\kappa}\right)$,

$N \approx \sqrt{2 \kappa x_{0}} \approx \sqrt{\frac{2 \kappa R_{+}}{b s}} \frac{1}{3^{1 / 4}}$,

$\frac{\mathcal{L}}{R_{+}} \approx b+\sqrt{3}+\delta_{1}$,

$\beta=\frac{1}{\sqrt{3}}+\frac{1}{b s}\left(\frac{\beta_{2}}{s} \frac{1}{3}-c\right)+O\left(b^{-2}\right)$,

$\mathcal{E}_{0}=X_{0} \approx \frac{2^{3 / 2}}{3^{3 / 4}} \sqrt{\frac{\kappa R_{+}}{b s}}$.

B. Modestly slow rotation

Now, one can check that, in contrast to the previous case, a finite $\beta$ is inconsistent with Eqs. (64) and (65). Instead, $\beta \sim b$ for large $b$. By trial and error approach, one can find that the suitable ansatz reads

$x=\frac{4}{9} R_{+} y a_{1}^{* 2}$,

where we introduced in this ansatz the dimensionless quantity

$a_{1}^{*}=R_{+}^{2} a_{1}$,

and the coefficient $\frac{4}{9}$ to facilitate comparison to the case of the Kerr metric (otherwise, this coefficient can be absorbed by $y$ ).

In doing so,

$\beta=\frac{4}{9} \beta_{0} a_{1}^{* 2} h(y)$,

$h \approx h_{1}-2 y$,

which is analog of (62), $\beta_{0}=b s$ according to (59). By definition, here $h \neq 0$. For the angular momentum we have from (9)

$\frac{\mathcal{L}}{R_{+}}=b+\beta_{+}=b+\frac{4}{9} \beta_{0} a_{1}^{* 2} h_{1}$.

Let us consider the main approximation with respect to the parameter $\varepsilon=\frac{4}{9} a_{1}^{* 2}$ and take into account that $|\beta| \gg 1$. Then Eq. (17) gives

$|\beta| \mathcal{L} \sqrt{2 \kappa x} a_{1} \approx \kappa \beta^{2}+\kappa x \frac{\mathrm{d} \beta^{2}}{\mathrm{~d} x}$. 
Equation (18) reads

$\mathcal{L}^{2} a_{1}^{2} \approx 2 \frac{\mathrm{d} \beta^{2}}{\mathrm{~d} x} \kappa+\kappa x \frac{\mathrm{d}^{2} \beta^{2}}{\mathrm{~d} x^{2}}$,

where now, with a given accuracy,

$\frac{\mathrm{d} \beta^{2}}{\mathrm{~d} x}=\frac{4}{R_{+}} \beta_{0}^{2} \varepsilon\left(2 y-h_{1}\right)$,

$\frac{\mathrm{d} \beta^{2}}{\mathrm{~d} x^{2}}=\frac{\beta_{0}^{2}}{R_{+}^{2}} \frac{\mathrm{d} h^{2}}{\mathrm{~d} y^{2}}=\frac{8 \beta_{0}^{2}}{R_{+}^{2}}$.

Substituting $\frac{\mathcal{L}}{R_{+}} \approx b$ into (193) and (194) and assuming $h<0$, one finds the system of two equations

$6 y-h_{1}=3 \delta \sqrt{y}$,

$h_{1}=3 y-\frac{9}{16} \delta^{2}$,

$\delta=\frac{1}{s \sqrt{2 \kappa R_{+}}}$. This system can be solved easily. There are two roots here but only one of them satisfies the condition $h \neq 0$ :

$y_{0}=\frac{\delta^{2}}{16}, \quad h_{1}=-\frac{3}{8} \delta^{2}, \quad h\left(y_{0}\right)=-\frac{\delta^{2}}{2}$.

(For $\beta>0$, one can obtain the equation $6 y-h_{1}=-3 \delta \sqrt{y}$ but in combination with (198) it would give $y<0$, which is unacceptable since outside the horizon we should have $y>0$. Thus this case should be rejected.)

Then, using (188), (192), and (12), we find the results (93)-(97).

\section{References}

1. D. Pugliese, H. Quevedo, R. Ruffini, Phys. Rev. D 84044030 (2011). arXiv:1105.2959

2. D. Pugliese, H. Quevedo, R. Ruffini, Phys. Rev. D 88024042 (2013). arXiv: 1303.6250
3. N.I. Shakura, R.A. Sunyaev, Astron. Astrophys. 24, 337 (1973)

4. Don N. Page, Kip S. Thorne, Astrophys. J. 191, 499 (1974)

5. J.M. Bardeen, W.H. Press, S.A. Teukolsky, Astrophys. J. 178, 347 (1972)

6. T. Jacobson, Class. Quantum Gravity 28, 187001 (2011). arXiv: 1107.5081

7. P. Pradhan, P. Majumdar, Eur. Phys. J. C 73, 2470 (2013). arXiv: 1108.2333

8. S. Ulbricht, R. Meinel, Class. Quantum Gravity 32, 147001 (2015). arXiv:1503.01973

9. O.B. Zaslavskii, Phys. Rev. D 92, 044017 (2015). arXiv: 1506.00148

10. M. Bañados, J. Silk, S.M. West, Phys. Rev. Lett. 103, 111102 (2009). arXiv:0909.0169

11. O.B. Zaslavskii, Pis'ma ZhETF 92, 635 (2010). arXiv:1007.4598. [JETP Lett. 9(2), 571 (2010)]

12. V.P. Frolov, Phys. Rev. D 85, 024020 (2012). arXiv: 1110.6274

13. T. Igata, T. Harada, M. Kimura, Phys. Rev. D 85, 104028 (2012). arXiv: 1202.4859

14. T. Harada, M. Kimura, Phys. Rev. D 83, 024002 (2011). arXiv: 1010.0962

15. O.B. Zaslavskii, Class. Quantum Gravity 29, 205004 (2012). arXiv: 1201.5351

16. O.B. Zaslavskii, Phys. Rev. D 84, 024007 (2011). arXiv: 1104.4802

17. M. Visser, Phys. Rev. D 46, 2445 (1992). arXiv:hep-th/9203057

18. A.A. Tursunov, M. Kološ, A.A. Abdujabbarov, B.J. Ahmedov, Z. Stuchlík, Phys. Rev. D 88, 124001 (2013). arXiv:1311.1751

19. O.B. Zaslavskii, Mod. Phys. Lett. A 29, 1450112 (2014). arXiv: 1403.6286

20. O.B. Zaslavskii, Mod. Phys. Lett. A 30, 1550027 (2015). arXiv: 1407.5440

21. A.A. Abdujabbarov, A.A. Tursunov, B.J. Ahmedov, A. Kuvatov, Astrophys. Space Sci. 343, 173 (2012)

22. R.M. Wald, Phys. Rev. D 10, 1680 (1974)

23. K.A. Bronnikov, O.B. Zaslavskii, Phys. Rev. D 78, 021501 (2008). arXiv:0801.0889

24. K.A. Bronnikov, O.B. Zaslavskii, Class. Quantum Gravity 26, 165004 (2009). arXiv:0904.4904

25. A.N. Aliev, D.V. Gal'tsov, Sov. Phys. Usp. 32, 75 (1989)

26. V.P. Frolov, A.A. Shoom, Phys. Rev. D 82, 084034 (2010). arXiv: 1008.2985

27. A.A. Grib, YuV Pavlov, O.F. Piattella, Int. J. Mod. Phys. A 26, 3856 (2011). arXiv: 1105.1540

28. F.J. Ernst, J. Math. Phys. 17, 54 (1976)

29. D.V. Gal'tsov, V.I. Petukhov, Sov. Phys. JETP 47, 419 (1978)

30. F.J. Ernst, W.J. Wild, J. Math. Phys. 17, 182 (1976) 